\title{
Hydrophobicity Classification of Polymeric Insulators Based on Embedded Methods
}

\author{
Zhengcheng Donga*, Yanjun Fang ${ }^{a}$, Xianpei Wang ${ }^{b}$, Yu Zhao ${ }^{b}$, Quande Wang $^{b}$ \\ ${ }^{a}$ School of Power and Mechanical Engineering, Wuhan University, Wuhan, 430072 China \\ ${ }^{b}$ School of Electronic Information, Wuhan University, Wuhan, 430072 China
}

Received: April 3, 2014; Revised: January 23, 2015

\begin{abstract}
Hydrophobicity is an important parameter to characterize electrical properties of insulated materials. Therefore, it is an urgent task to develop on-line instruments to identify the hydrophobicity of insulated material's surface conveniently, quickly and accurately. For this purpose, a novel evaluation system with image processing and decision tree is proposed which is based on embedded platform. For obtaining satisfactory results, we first propose a mixed image segmentation method to overcome the complex conditions outside, concerning non-controlled illumination, nonstandard surfaces and unfixed shooting angle. Then we adopt four new characteristic parameters to describe the image of each sample. Finally, a classification method based on MultiBoost decision tree is conducted which synthesizes the merits of both AdaBoost and Wagging algorithm. Results indicate the procedures can be applied in the DSP (Digital Signal Processor) platform perfectly and better results can be obtained than those did in our previous study or that of some other research.
\end{abstract}

Keywords: polymeric insulators, hydrophobicity, image processing, characteristic parameters, MultiBoost decision tree

\section{Introduction}

With the increasing demands of power, the security problem of power grid is becoming more and more serious. Insulator, as the key component of the system, is related to the safety of the entire grid. In recent years, polymeric insulators have been widely used in power supply and distribution systems because of good shatterproof nature, light weight, superior mechanical property and low maintenance $\operatorname{cost}^{1-3}$. Insulator with hydrophobic surface has better electrical flashover characteristics than that with hydrophilic surface or glass. However, the hydrophobicity of polymeric insulators in service will degrade by many factors, such as pollution deposits, surface arcing, and aging. Therefore, it is necessary to find an effective method for determining the hydrophobic level of insulated material's surface.

According to the guide of IEC62073, three methods are given for the measurement of hydrophobicity, i.e. contact angle method, surface tension method and spray method ${ }^{4}$. The first two traditional laboratorial methods of measuring contact angles and surface tension are not practical in the field because requirements of well-defined experimental conditions can't be satisfied, such as fixed illumination, optimal view of a single water drop, or small-flat, horizontal samples ${ }^{5}$.

Oppositely, the spray method is widely used because of its simple and low requirement of equipments. As a pioneer work, the HC (Hydrophobicity Classification) method proposed by STRI (Sweden Transmission Research Institute) offers a simple procedure for obtaining a collective

*e-mail: dongzhengcheng@whu.edu.cn estimate of an insulating surface's hydrophobicity in the field which is regarded as the authoritative standard ${ }^{6-8}$. In this method, six hydrophobic classes from HC1 to HC6 are defined, according to the shape of waterdrops and the percentage of wet regions on the hydrophobic surface. The defined $\mathrm{HC} 1$ performs the highest hydrophobic surface, where only discrete and extremely circular waterdrops are formed. With the increase of $\mathrm{HC}$, the hydrophobicity declines gradually. When it approaches to HC4 or HC5, the insulator is becoming hydrophilic, which in turn can be interpreted as a warning sign.

Traditional HC method has some subjective drawbacks which requires skillful technicians and proper experimental time. Therefore, some objective measuring methods based on image processing and feature extraction are proposed, 5,9-22 such as fractal dimension, circular factor, goniometric measurement using Hough transformation, scaled entropy and histogram analysis, surface energy, and online hydrophobicity measurement methodology. However, only one or two characteristic parameters are adopted for classification in these methods which can't describe images comprehensively. Furthermore, researchers always focus on the improvement of methods, and there is still no research on embedded instruments for on-site measurement.

Therefore, an embedded system for measuring hydrophobicity named EIMHMS (Embedded Measuring System of Insulator Material Hydrophobicity) is designed by misjudging-cost in this paper. The methods used in EIMHMS are easily implemented, and this establishes the foundation for embedded measuring instruments. In 
EIMHMS, a series of processing procedures are proposed for better segmenting droplets which are suitable for embedded platform. Furthermore, in order to synthesize the characteristic parameters mentioned above, four typical parameters are proposed to depict the feature of each sample. Then a classifier based on MultiBoost decision tree $e^{23-27}$ is employed, and the generated "if-else" rules can operate without primary algorithm. In the end, promising results can be obtained in EIMHMS and all the procedures can be applied in embedded platform perfectly.

\section{Experimental Procedure}

\subsection{Equipment}

In our experiments, a digital camera with 14 million pixels and 25X optical zoom (Sony, W-315), a personal computer and a tiltable platform used for fixing samples are equipped. Furthermore, insulator specimens with different hydrophobic levels are needed, and each sample used in experiments is thin circular plate which is made of silicone rubber (SIR) with light red color, and has a thickness of $5 \mathrm{~mm}$ and a diameter of $190 \mathrm{~mm}$. The waterdrop patterns are produced by an ordinary spray bottle containing distilled water.

\subsection{Experiment}

For simulating actual conditions in the field, before our tests, some principles should be followed (see Figure 1)

1) All the images should be taken outside,

2) Each sample should be placed with a suitable height $H_{V}$ and angle $\alpha$,

3) There is a horizontal distance of $H_{H}=2 \mathrm{~m}-3 \mathrm{~m}$ between the camera and sample,
4) Any auxiliary spotlights are forbidden.

For obtaining samples with various HC, Thomazini et al. ${ }^{16,19,20}$ artificially change the hydrophobicity of specimen with spraying WIA (Water and Isopropyl Alcohol) solution at different concentrations (from 0 to $100 \%$ ). Although this method can make different levels of hydrophobicity with only one or two specimens, the images obtained are extremely standard which can't present the actual situations of the insulator's surface. Therefore, we adopt another approach for obtaining samples with spraying water on insulators of different HC. First, more than 140 insulators with different hydrophobicity (the number of specimens with HC1-HC6 is respectively 21, 22, 25, 28, 21, and 24) are provided by WHVRI (Wuhan High Voltage Research Institute). Each specimen is labeled for a $\mathrm{HC}$ which has been defined by various tests, such as DDT (Dynamic Drop Test) and STM (Surface Tension Method), and these insulators can be used as the standard specimens. The experiment consists of the following steps:

Step1. Place the tiltable platform at the height of $2 \mathrm{~m}$ from the ground,

Step2. Fix the sample on the top of the platform and make it titled by $30^{\circ}$ from the horizontal,

Step3. Spray pre-prepared distilled water on the surface of the fixed sample with spray bottle,

Step4. When the camera and droplets approach to the steady statue, photograph the spraying image with camera at the horizontal distance of $3 \mathrm{~m}$,

Step5. Repeat steps above until the images of all the samples are obtained.

After getting all the images, image processing and classification will be followed. For subsequent analysis, images are transferred to a personal computer with USB

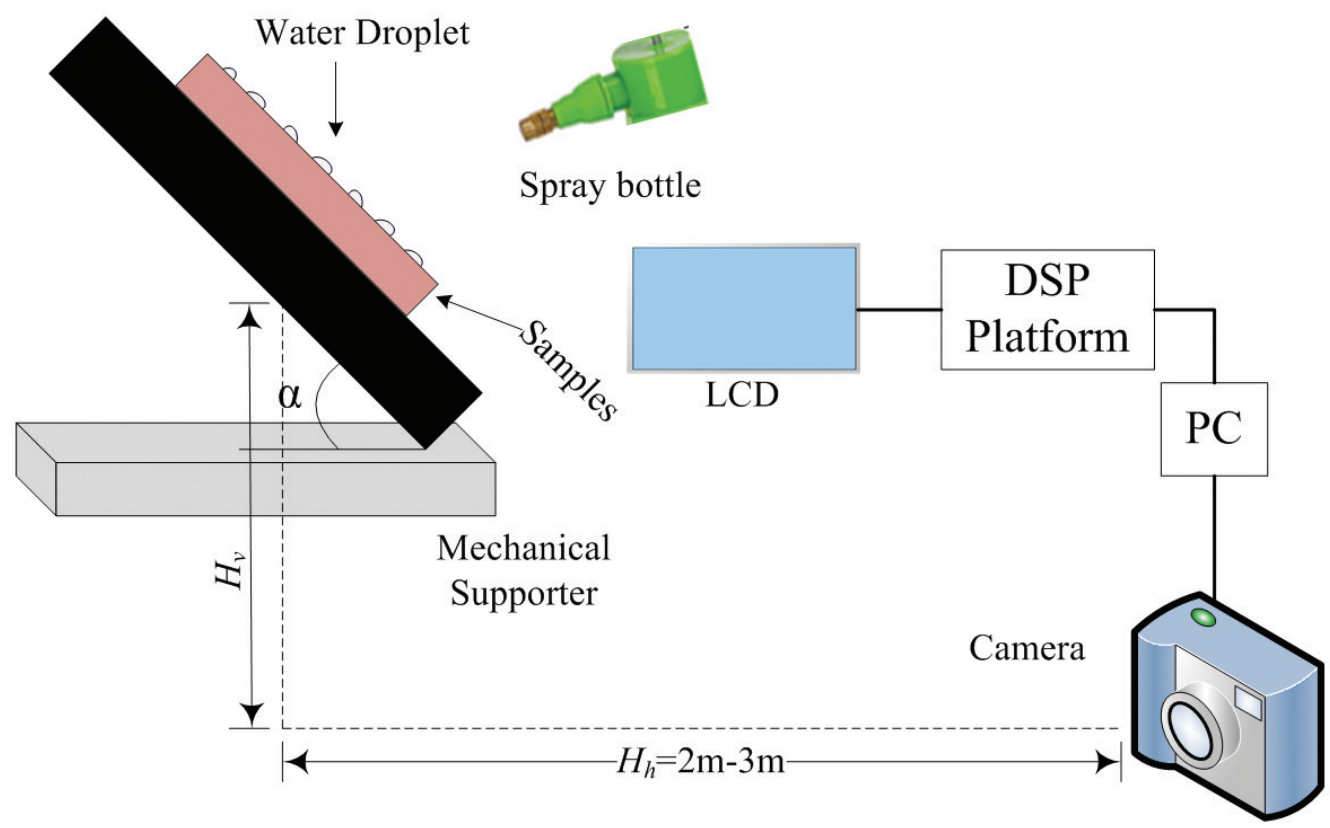

Figure 1. Illustration of equipments. 
(Universal Serial Bus) interface. The main software tool for computation is the Matlab v7.11 and its image processing toolbox v7.1, which also provides a simple user-friendly environment for image analysis and GUI (Graphical User Interface) design. PC used for experiments is a Dell computer with a $3.0 \mathrm{GHz} \mathrm{CPU}$ and $4 \mathrm{~GB}$ ram. Furthermore, a DSP platform with TMSDM6446 processer is also set up for testing procedures. Most codes in experiments are programmed to DSP by CCSLink toolbox (Matlab Link for Code Composer Studio).

\section{Image Processing Methods}

More than 140 images are made during the course of experiments. In order to exclude the edge of insulator plate, only the central part of each collected image is used, i.e. a rectangular region of $200 \times 200$ pixels. Before Image processing, all the RGB images should be transformed into gray images to reduce the amount of calculation. Some original images with various HC are shown in Figure 2.

It is difficult to recognize waterdrops from images because the color of insulators is various and the background of images is much complex. Furthermore, water transparency leads to smaller gray difference, and light reflection leads to fuzzy boundary. In order to extract intact droplets and operate on the DSP platform, simple and appropriate image processing methods should be proposed. Here we propose an adaptive threshold segmentation method based on canny operator (COATS) which can produce better results than single method. To reduce elapsed time, we introduce the integral image to replace the original image. In the end, binary image optimization based on mathematical morphology is conducted.

\subsection{Adaptive threshold segmentation}

An integral image is a tool that can be used whenever we have a function from pixels to real numbers $f(x, y)$, and we wish to compute the sum of this function over a rectangular region of the image $e^{26-28}$. If we need to compute the sum over multiple overlapping rectangular windows, we can use an integral image and achieve a constant number of operations per rectangle with only a linear amount of preprocessing.

$$
I(x, y)=f(x, y)+I(x-1, y)+I(x, y-1)-I(x-1, y-1)
$$

Where $I(x, y)$ represents the integral image, $f(x, y)$ represents the total pixels of a rectangular region. With the integral image, the sum of the function for any rectangle with upper left corner $\left(x_{1}, y_{1}\right)$, and lower right corner $\left(x_{2}, y_{2}\right)$ can be computed in constant time using the following equation

$$
\sum_{y=y_{1}}^{y_{2}} f(x, y)=I\left(x_{2}, y_{2}\right)-I\left(x_{2}, y_{1}-1\right)-I\left(x_{1}-1, y_{2}\right)+I\left(x_{1}-1, y_{1}-1\right)
$$

The main idea in adaptive threshold algorithm is that each pixel is compared to an average of its surrounding pixels. If the value of the current pixel is $t$ percent lower than the average then it is set to black, otherwise it is set to white.
With the integral image, we compute the average of an $s \times s$ window of pixels centered around each pixel, and the pseudo-code is shown below ${ }^{28}$.

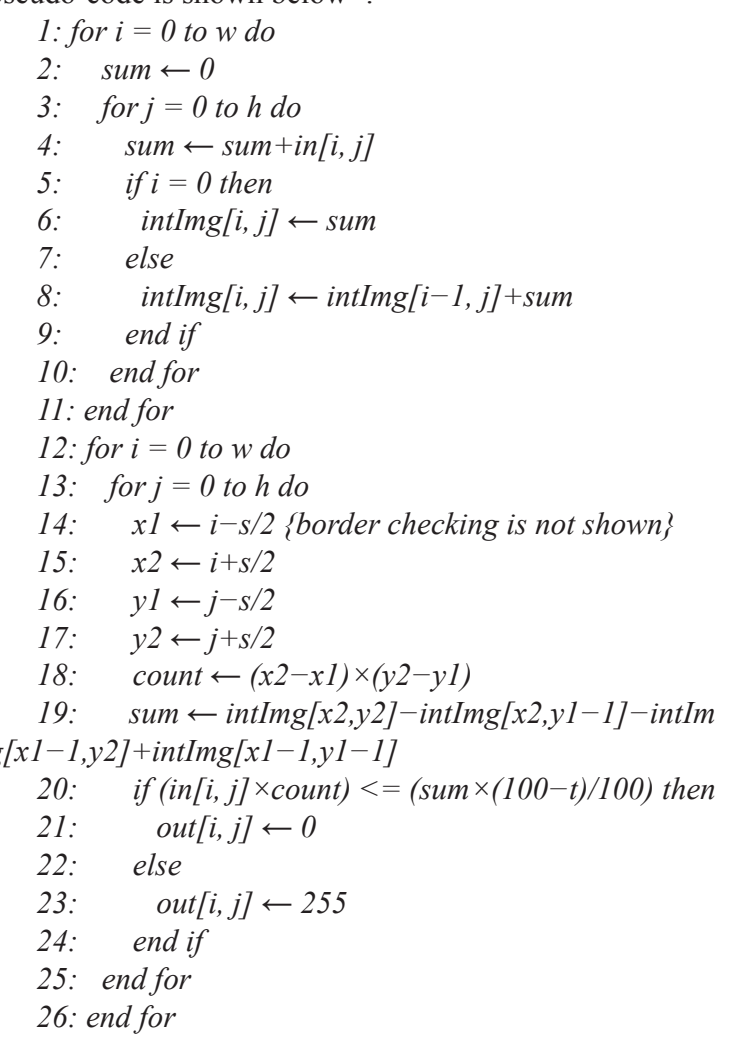

\subsection{Improved canny operator}

Canny edge detection algorithm ${ }^{29}$ is one of the most commonly used image processing algorithms on embedded platform with its easy programming, excellent performance and the three criteria ${ }^{30}$. However, when applying Gaussian filter, it will cause the loss of edge, and with the influence of shadow, it will sometimes provide false results. Therefore, an improved canny operator based on droplets is proposed:

1) Conduct traditional canny operator and obtain the edge image $E(i, j)$. Then label all the isolated lines as $L_{l}, L_{2}, \ldots, L_{N}$. If $L_{I}$ is a closed curve, we consider $L_{I}$ as the real edge; otherwise skip to step 2).

2)For an open curve $L_{J}$, we will conduct further diagnose. First, label the two endpoints $a$ and $b$ of $L_{s}$, and select $n$ points between $a$ and $b$, i.e. $d_{1}, d_{2}, \ldots, d_{N}$. Second, respectively calculate the tangent's oblique angle $A_{1}, A_{2}, \ldots, A_{N}$ of each point in $L_{J}$. In the end, calculate the difference $\delta_{A}$ between the maximum $A_{M A X}$ and minimum $A_{M I N}$ of $A_{I}$. If $\delta_{A}>\pi$, we consider $L_{J}$ is real edge of droplets; otherwise $L_{J}$ is the false edge produced by shadow and should be cast out.

3) With all the procedures above, results can be obtained, and denoted by $E E(i, j)$.

\subsection{COATS method}

In order to obtain promising results, more details about waterdrops and edges should be applied. With results, we find that the canny operator is sensitive to noises and illumination, and the adaptive threshold method causes 


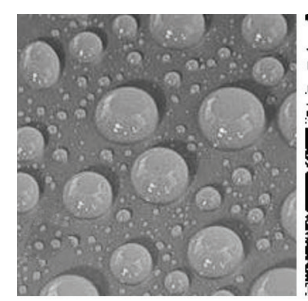

(a1)

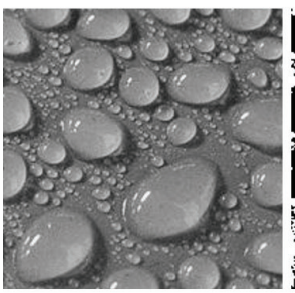

(a2)

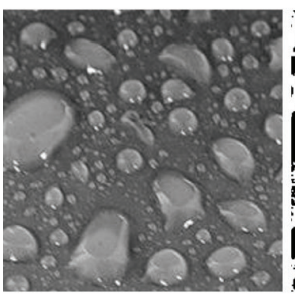

(a3)

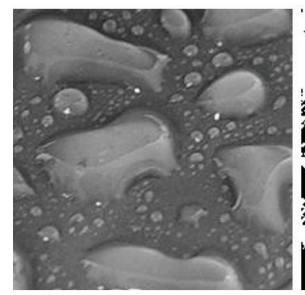

(a4)

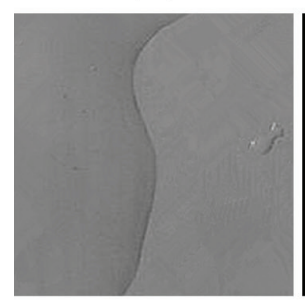

(a5)

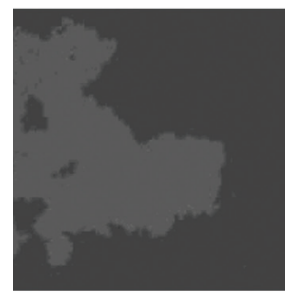

(a6)

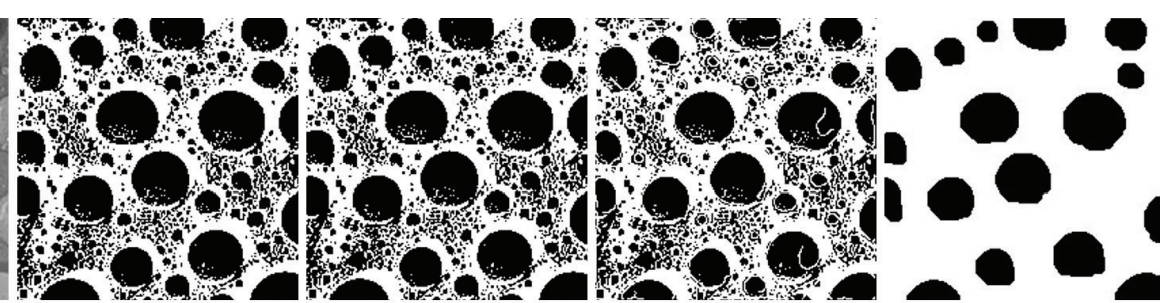

(b1) (c1) (d1)

(e1)

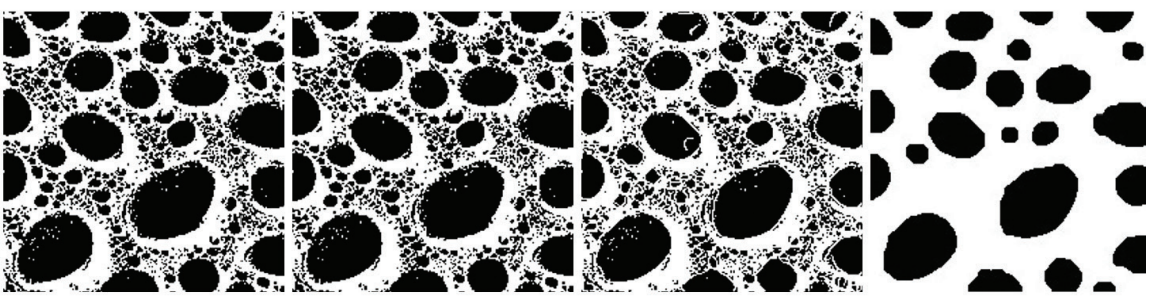

(b2)

(c2)

(d2)

(e2)

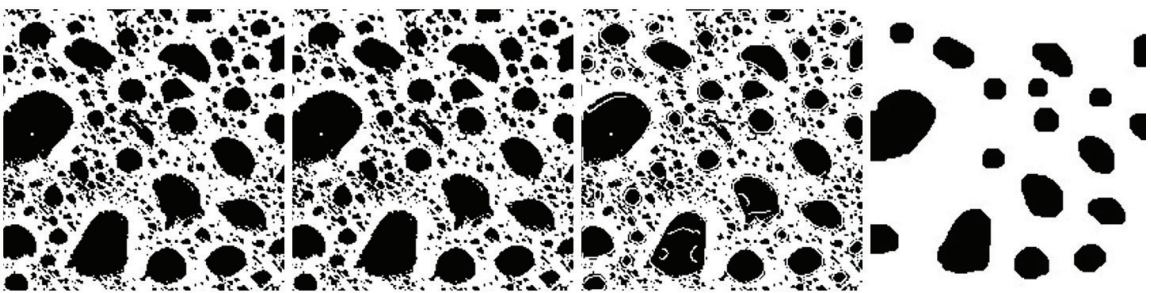

(b3)

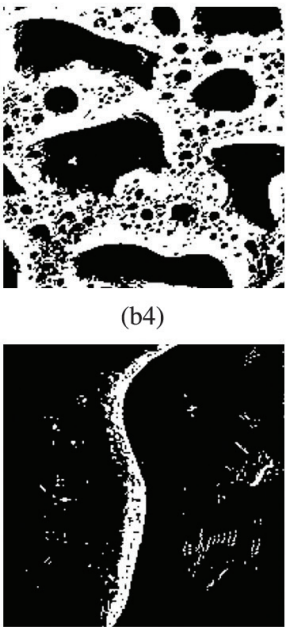

(b5)

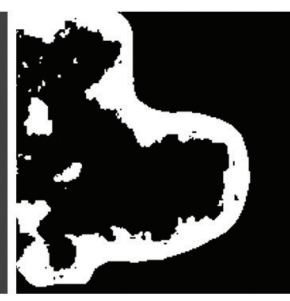

(b6) (c3)

$+4$

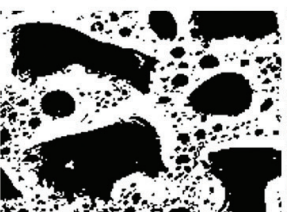
if $x^{3}$

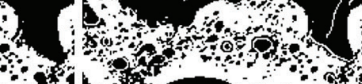

(d3)

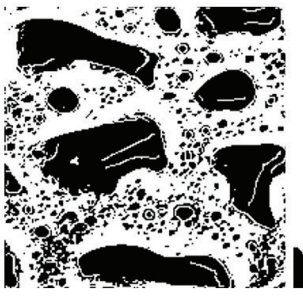

(d4)

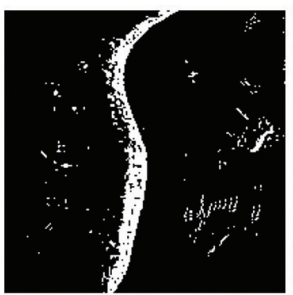

(c5)

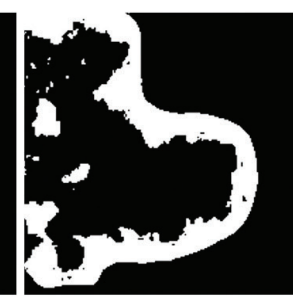

(c6)

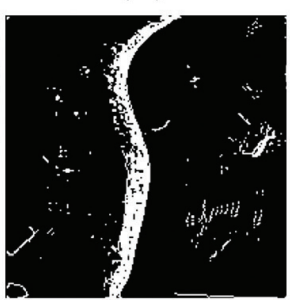

(d5)

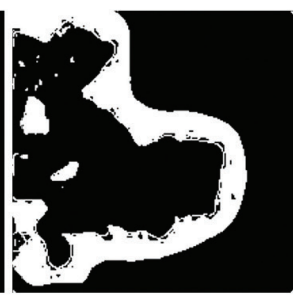

(d6)

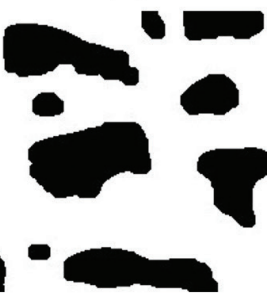

(e4)

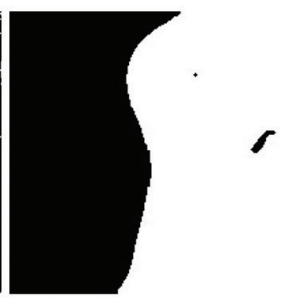

(e5)

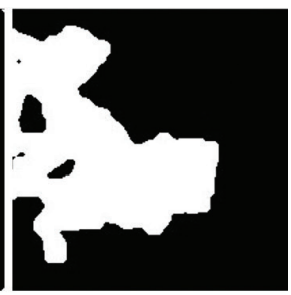

(e6)

Figure 2. Image processing results. a1)-a6) represent the original images from HC1-HC6, b1)-b6) represent the results of the adaptive threshold method from HC1-HC6, c1)-c6) represent the results of the COATS method from HC1-HC6, d1)-d6) represent the final results of mathematical morphology from HC1-HC6. Average elapsed time is 0.922 seconds on PC and 2.3 seconds on DSP. 
fuzzy periphery of each segmentation region. Therefore, we propose a mixed method combining the results of two methods. Considering the operational capability and programming complexity of DSP, the final results are obtained by adding these two images simply which are proved to be good enough.

\subsection{Binary image optimization based on mathematical morphology}

In order to remove noises and useless points which are still in results, we adopt a series of morphological operations $^{31}$ :

1) Apply erosion and morphological reconstruction operations to remove small pixels and keep the original shape of the rest pixels,

2) Apply close operation to fill some narrow breaks in the droplets.

3) Eliminate the rest of small droplets and noises with opening operation.

\subsection{Results and analysis}

All the procedures above are applied on DSP platform, and the results in Figure 2 are operated on DSP platform and displayed in Matlab window. Furthermore, some results on LCD of DSP platform are shot by camera (see Figure 3).

The adaptive threshold method with integral image consumes less time than some other methods (tests in our other experiments) and performs better for the image with uneven illumination. Because of its easy implementation, it can operate on the embedded equipment perfectly. As shown in Figure 2b, although approximate shapes of droplets with different hydrophobicity are segmented, there are still some noises, conglutination and small useless droplets. Therefore, more information should be applied for further extraction, and we conduct an improved canny operator. However, although some accurate edges are obtained, there are still some redundant pixels (see Figure 2c), and with mathematical morphology operations, these pixels will be removed completely (see Figure $2 \mathrm{~d}$ ).

As shown in Figure 2d, all the procedures of image processing have been completed, and most droplets are extracted integrally. For the low hydrophobic images, such as $\mathrm{HC} 5$ and HC6, in which there is only one or two big water film parts, are easy to be segmented because of the strong contrast. However, it is difficult to recognize which region is wet. As shown in Figure $2 b$ and $2 c$, there are more noises in the unwetted region than that of the wet region, and we can distinguish by using this criterion.

As the samples are labeled by experiments and experts, the errors due to images are derived from the inaccurate $\mathrm{HC}$ which may result in misjudgements in the end. However, these samples are tested for many methods, and the WHVRI has also conducted verification tests, and we consider that the errors can be ignored for classification. Furthermore, the small quantity of samples can result in inaccurate classification model which may reduce the accuracy of classifier (the analysis will be elaborated in Sec. 6).

\section{Characteristic Parameters Extraction}

For classifying different hydrophobic levels, some characteristic parameters should be given for depicting each image. While there are many attributions proposed by experts, such as fractal dimension, circular factor, the largest shape factor and so on. To synthesize the advantages above, this paper adopts four parameters improved by our previous work.

\subsection{Characteristic parameters}

Let $N$ be the number of droplets recognized, $S_{I}, C_{I},\left(x_{P} y_{P}\right)$ be area, perimeter and center of bound rectangle of droplet $i(0 \leq i \leq N)^{32}$.

1) Cover: Cover is the ratio of areas covered by water to areas not covered by water

$\operatorname{cov}$ er $=\sum_{i=1}^{N} S_{i} / L W$

Cover is one of most common parameters used for judging hydrophobicity of materials and is an important characteristic parameter which represents the overall hydrophobicity of material's surface.

2)Dis_uni:Dis_uni describes the uniformity of distribution in nine equal regions of a spraying image. The more evenly the waterdrops distribute, the bigger the dis_uni becomes.

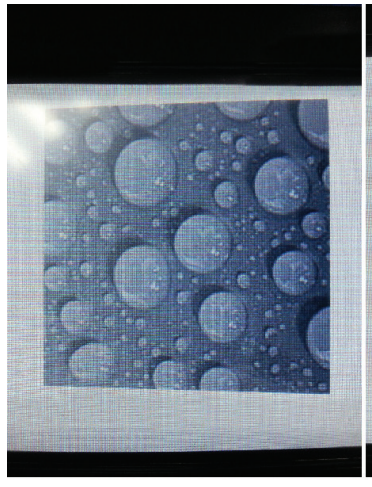

(a)

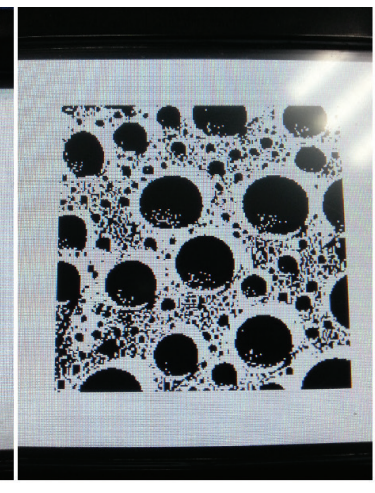

(b)

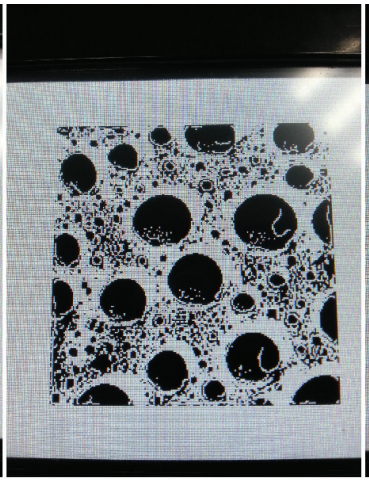

(c)

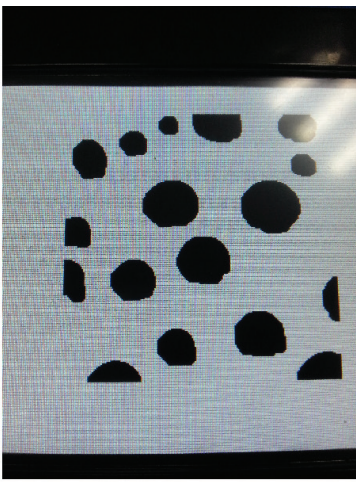

(d)

Figure 3. Results on LCD of the DSP platform. a)-d) represent the original image, threshold segmentation result, COAT result and final result. 
dis_uni $=-\sum_{\text {row }=0}^{2} \sum_{\text {col }=0}^{2} \frac{\mathrm{n}(\text { row }, \text { col })}{\mathrm{N}} \ln \left(\frac{\mathrm{n}(\text { row }, \text { col })}{\mathrm{N}}\right)$

Where

$\mathrm{n}($ row, col $)=\mid\left\{\mathrm{i} \mid \operatorname{col} \times \frac{\mathrm{L}}{3} \leq \mathrm{x}_{\mathrm{i}} \leq(\operatorname{col}+1) \times \frac{\mathrm{L}}{3} ;\right.$ row $\times \frac{\mathrm{L}}{3} \leq \mathrm{y}_{\mathrm{i}} \leq($ row +1$\left.) \times \frac{\mathrm{L}}{3}\right\} \mid$

The computation of Dis_uni is essentially in calculating

the shannon entropy of water distribution. The whole image is divided into nine zones $c_{1}, c_{2}, \ldots, c_{K}(k=1$, $2, \ldots, 9)$, and we separately calculate the probability $p_{I}=n(\mathrm{row}, \mathrm{col}) / N$ of $c_{K}$ which means the probability of droplets falling into $c_{K}$, and then get its entropy. The bigger entropy means evenly distributed droplets and better hydrophobicity.

3)Area_uni:Area_uni describes the uniformity of areas covered with water.

area_uni $=\frac{\sum_{i=1}^{n}\left(S_{i}-\bar{S}\right)}{N \bar{S}}$, and $\bar{S}=\frac{\sum_{i=1}^{N} S_{i}}{N}$

Area_uni represents the size of droplets from the side, and is equivalently to calculate the area deviation of each droplet and the mean area. Bigger deviation indicates bigger Area-uni and worse evenness of distribution. To some extent, it also tells the difference between droplets and water films of some insulators with lower hydrophobic levels. Because the area of water film is bigger than uniform droplet a lot, the value of Area_uni is bigger when there are some water films.

4)Round_de:Round_de is the average round degree of all droplets.

round_de $=\frac{\sum_{i=1}^{N} \frac{4 \pi S_{i}}{C_{i}^{2}}}{N}$

$4 \pi S_{I} / C_{i}^{2}$ is the formula of calculating roundness of irregular circle. In the equation above, $4 \pi S_{I} / C_{i}^{2}$ is used for calculating the area of standard circle, and the roundness can be obtained with dividing by the real area $S_{I}$. Round_de represents the shape of droplets, and it is closer to ideal circle when Round_de approaches to 1.

\subsection{Tests of geometrical independence}

As we know, a good characteristic parameter should have the geometrical independence. Therefore, we make rotation and scale transformation on spraying images with $\mathrm{HC} 1-\mathrm{HC} 6$, and observe the changes of Cover, Dis_uni, Area_uni, and Round_de in the case of geometric transformation.

1) Tests of Cover

Make rotation and scale transformation, and observe the change of Cover. As shown in Figure 4, 4a is the test of scale transformation, and $4 \mathrm{~b}$ is the test of rotation transformation.
As shown in Figure 4a, Cover has obvious change only when the image narrows down to $0.1-0.3$ of the original image, and Cover is inaccurate when the rate approaches 0.1. As shown in Figure 4b, Cover almost has no change when making the rotation tests. So we can conclude that the parameter Cover has good geometrical independence.

2) Tests of Dis_uni

Make rotation and scale transformation, and observe the change of Dis_uni. As shown in Figure 5, 5a is the test of scale transformation, and $5 b$ is the test of rotation transformation.

As shown in Figure 5a, Dis_uni has obvious change only when the image narrows down to $0.1-0.3$ of the original image, and Dis_uni is inaccurate when the rate approaches 0.1. As shown in Figure 5b, Dis_uni almost has no change when making the rotation tests. So we can conclude that the parameter Dis_uni has good geometrical independence.

3) Tests of Area_uni

Make rotation and scale transformation, and observe the change of Area_uni. As shown in Figure 6, 6a is the test of scale transformation, and $6 \mathrm{~b}$ is the test of rotation transformation.

As shown in Figure 6a, Area_uni has obvious change only when the image narrows down to $0.1-0.3$ of the original image, and Area_uni is inaccurate when the rate approaches 0.1. As shown in Figure 6b, Area_uni almost has no change when making the rotation tests. So we can conclude that the parameter Area_uni has good geometrical independence.

4) Tests of Round_de

Make rotation and scale transformation, and observe the change of Round_de. As shown in Figure 7, 7a is the test of scale transformation, and $7 \mathrm{~b}$ is the test of rotation transformation.

As shown in Figure 7a, Round_de of HC5-HC6 has obvious increase when the image narrows down to 0.1-0.3 of the original image, and Round_de of HC1-HC4 has obvious decline when the rate approaches 0.1-0.2. As shown in Figure 7b, Round_de almost has no change when making the rotation tests. So we can conclude that the parameter Round_de has good geometrical independence.

The parameters above are selected from lots of attributions of the spraying image. They are all independent to the real size and angle of images that is convenient for classification. The four parameters of samples are shown in Figure 8, and we can find that Dis_uni has poor distinguish ability and on the contrary the other three parameters are better for classification.

\section{Classification Based on MultiBoost Decision Tree}

After getting attributions of all spraying images with different hydrophobic levels, classification will be employed in the end. In our experiments, both the PLSR (Partial Least-Square Regression) method ${ }^{33}$ based on mathematical model and the decision tree method based on machine learning are carried out. Compared with results, we conclude that there is no obvious mathematical relation between the characteristic parameters and hydrophobic levels adopted in this paper. In order to develop embedded equipment 

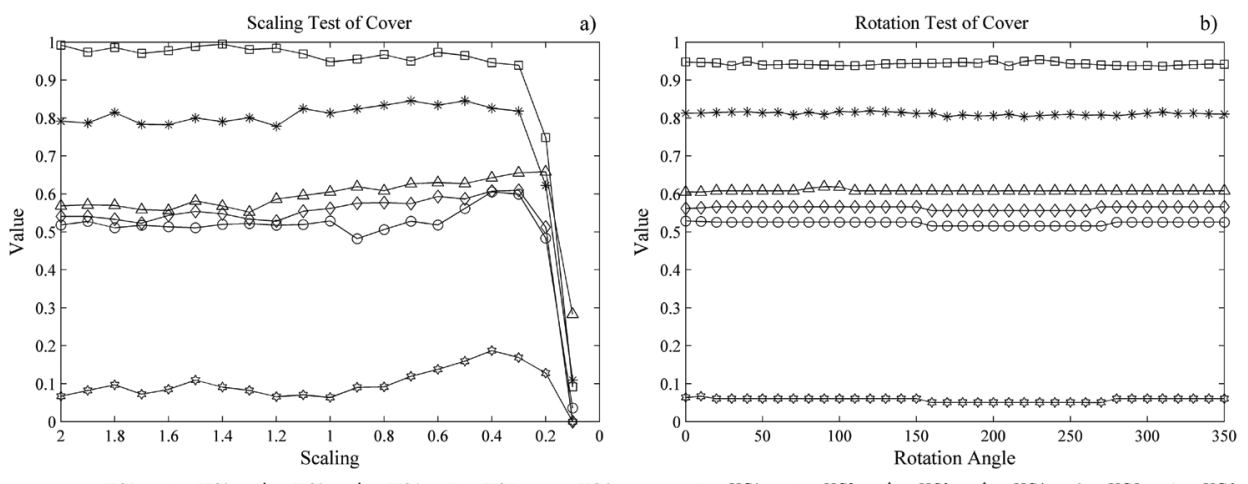

Figure 4. Tests of Cover with various hydrophobic levels. a) Scale transformation, b) Rotationtransformation.
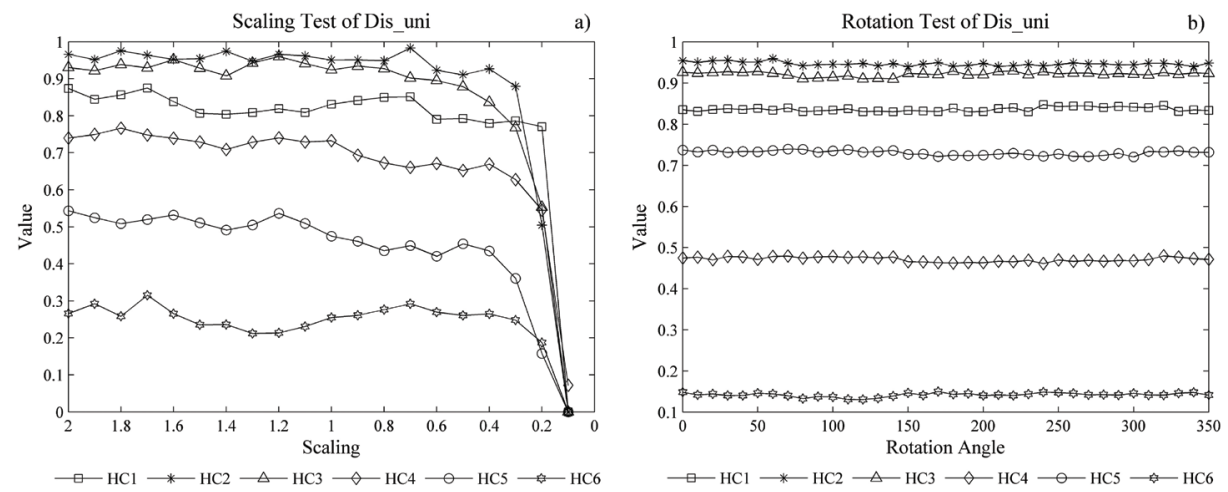

Figure 5. Tests of Dis_uni with Various Hydrophobic Levels. a) Scale Transformation, b) Rotation Transformation.
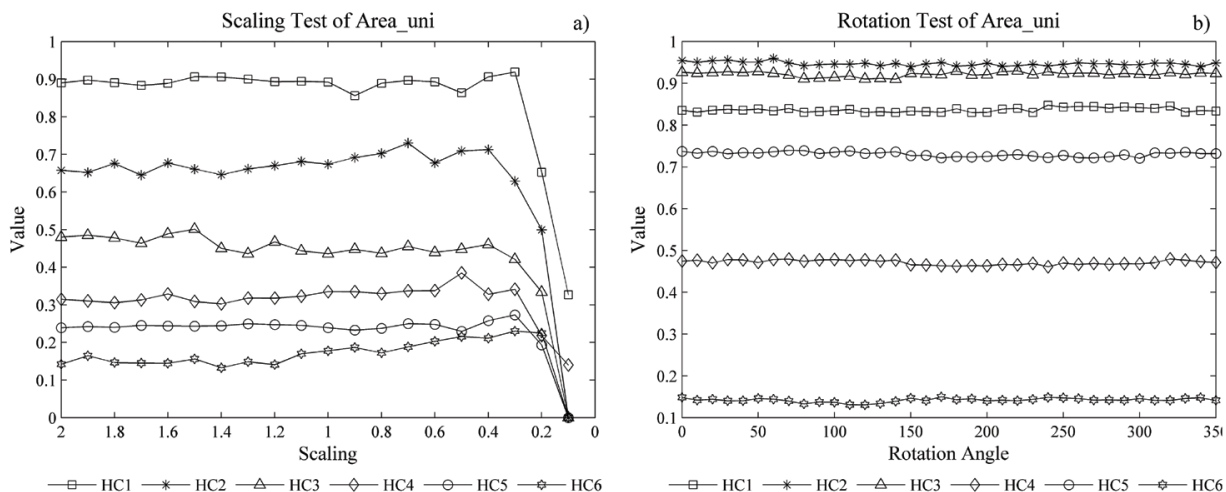

Figure 6. Tests of Area_uni with Various Hydrophobic Levels. a) Scale Transformation, b) Rotation Transformation.
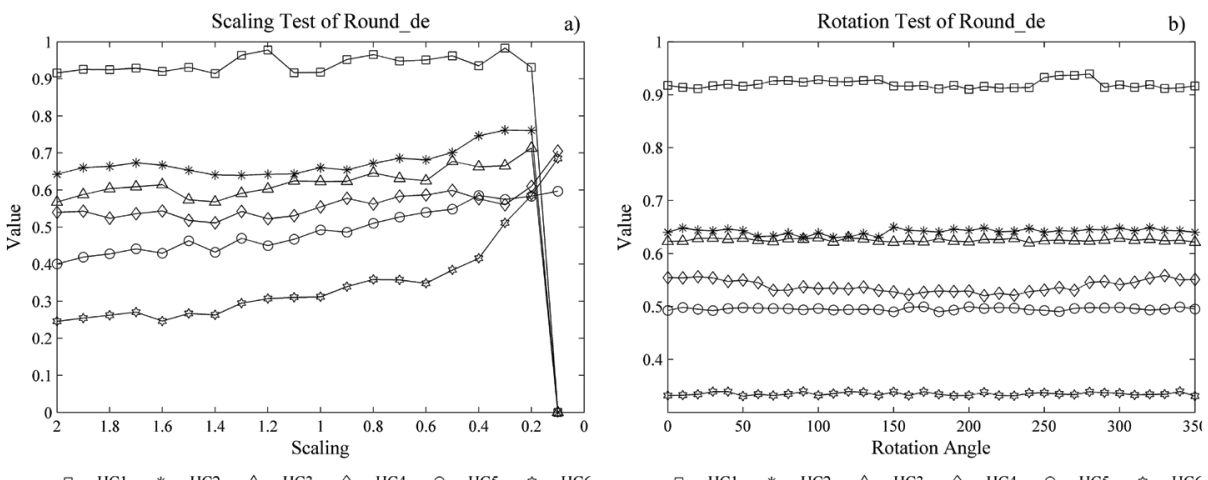

Figure 7. Tests of Round_de with Various Hydrophobic Levels. a) Scale Transformation; b) Rotation Transformation. 
for measuring hydrophobicity, simple and easy methods for classification should be adopted. The "if-else" rules of decision tree are fit for running in MCU (Micro Control Unit) with low operation speed.

The training and classification steps of decision tree induction are simple and fast which can be applied to any domain of data distribution. However, simple classifier can't meet the needs of error yet, and the committee learning algorithm is proposed for classification. Decision committee learning has demonstrated spectacular success in reducing classification errors generated by learned classifiers. These techniques develop a classifier in the form of a committee of subsidiary classifier. The committee members are applied to a classification task and their individual outputs are combined to create a single classification from the committee as a whole. This combination of outputs is often performed by majority vote. Examples of these techniques include classification ensembles formed by Bagging, AdaBoost, and Wagging 23,24 .

\subsection{MultiBoost decision tree}

Two decision committee learning approaches, AdaBoost and Bagging, have received extensive attention. Both AdaBoost and Bagging are generic techniques that can be employed with any base classification techniques. They operate by resampling selectively from the training data to generate derived training sets to which the base learner is applied. A number of studies comparing AdaBoost and Bagging suggest that AdaBoost and Bagging have quite different operational profiles. In general, it appears that

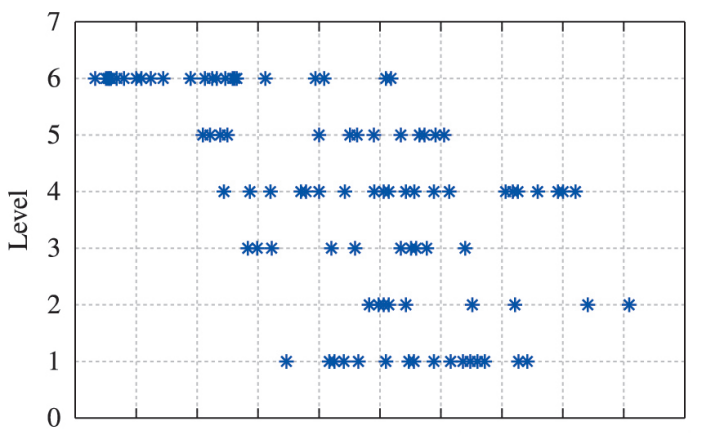

$\begin{array}{lllllllllll}0.00 & 0.06 & 0.12 & 0.18 & 0.24 & 0.30 & 0.36 & 0.42 & 0.48 & 0.54 & 0.60\end{array}$ Cover

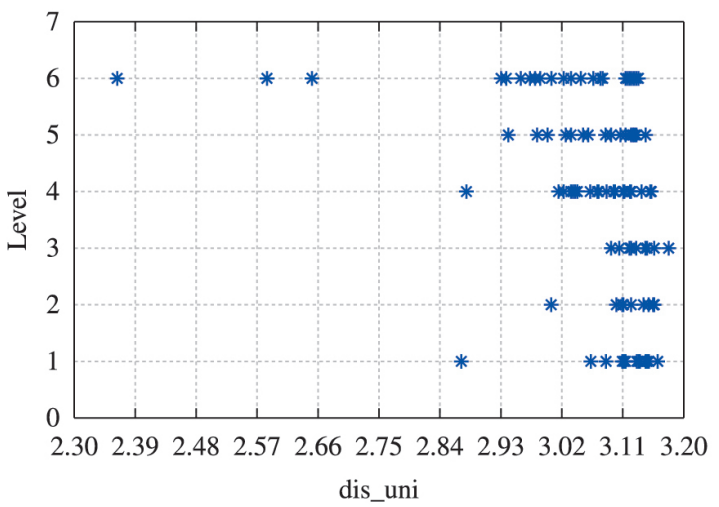

Bagging is more consistent, and the frequency to increase errors of the base learner is less than AdaBoost does. However, AdaBoost appears to have greater average effects, and has substantially larger error reductions than Bagging does on average. It is confirmed that AdaBoost reduces both bias and variance while Bagging and Wagging have little effect on bias and greater effect on variance ${ }^{25}$. MultiBoost (Combining Boosting and Wagging) is shown to achieve most of AdaBoost's superior bias reduction coupled with most of Bagging's superior variance reduction.

\subsection{Result and analysis}

Given the theories and experiments above, a MultiBoost tree based on C4.5 is adopted for our classification. Results of training and testing are provided by DSP platform with "if-else" rules, and $k$-fold cross validation is applied by Matlab (see Figure 9).

Firstly, because of the limited number of samples, we conduct $k$-fold cross validation method to verify the rules of decision tree, which divides the full data set into $k$ subsets. When modeling, only $k-1$ subsets are used, and the remaining subset is used for validation data to verify the model. In this case, experiments will be repeated for $k$ times, and there will be a predicted value in the end. The advantage of this approach is that it repeatedly uses random subsets for training and validation at the same time. $K$-fold cross validation is used for training and validation with the small data set, and it also can test the stability of model. Furthermore, training and testing experiments are also employed, which divide the full data set into two subsets,
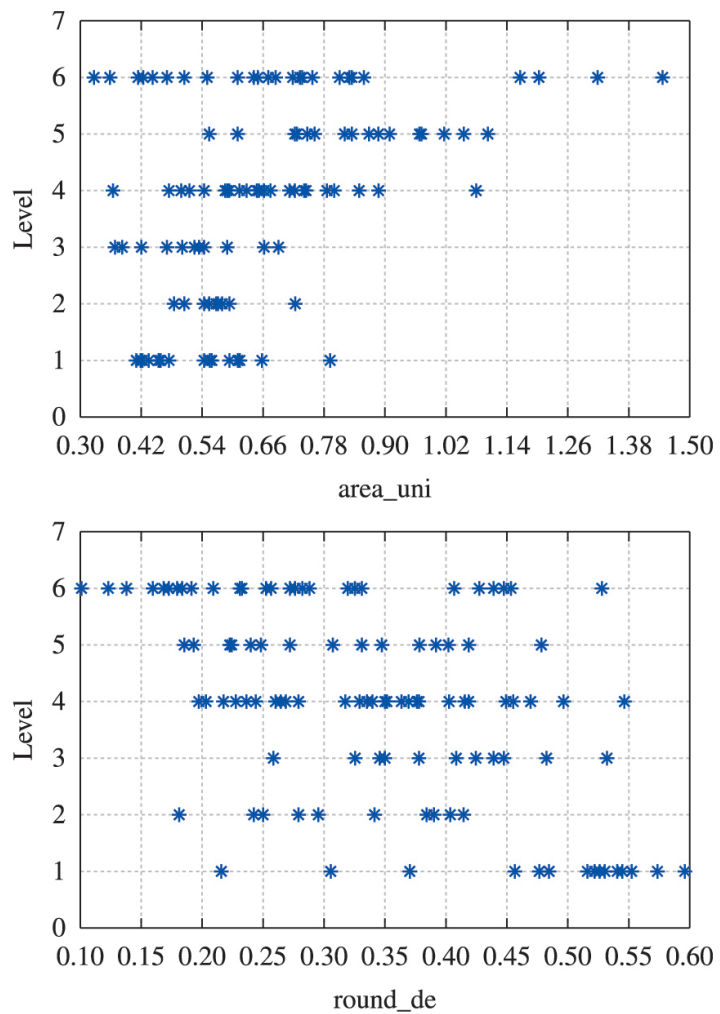

Figure 8. The characteristic parameters of samples with various hydrophobic levels. 


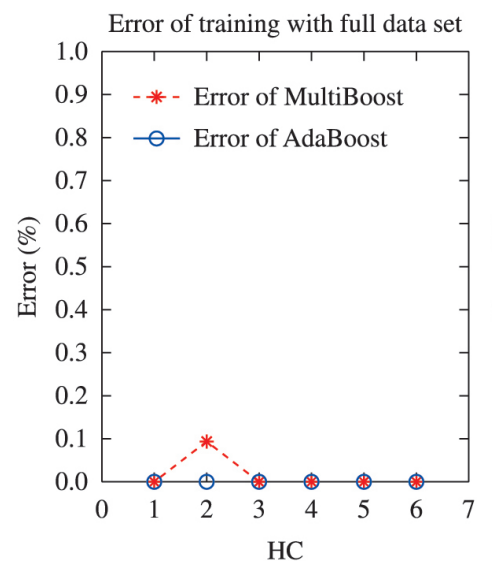

(a)

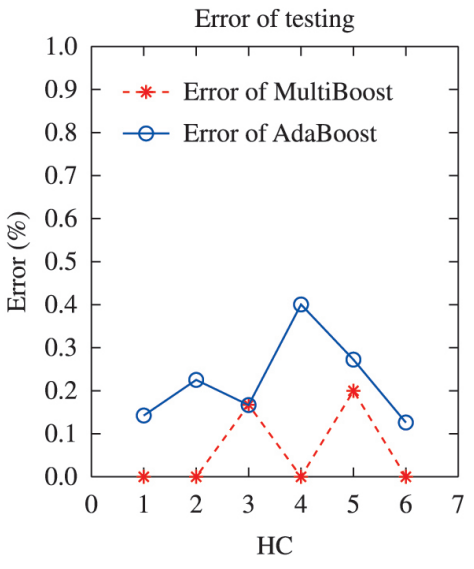

(b)

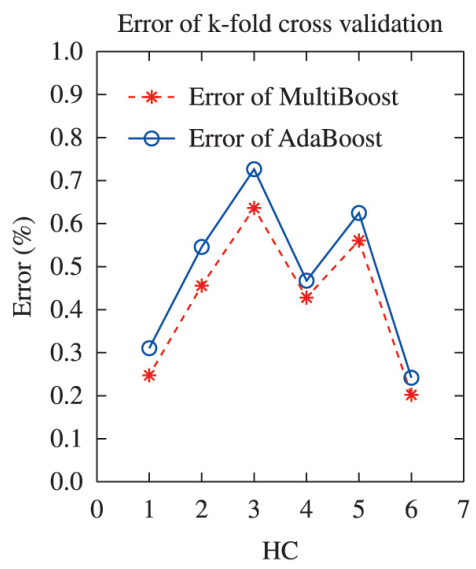

(c)

Figure 9. Errors (\%) of AdaBoost and MultiBoost algorithm. a) Errors with training subset, b) Errors with testing subset, c) Errors with $k$-fold cross validation method.

i.e. training data and testing data. As shown in Figures 9a and c, the error (\%) of MultiBoost is less than AdaBoost algorithm. Because of small data set, only a few samples are applied for testing, and the error (\%) is relatively large.

With results, we can conclude that both AdaBoost and MultiBoost methods can achieve a higher precision with the full data set, and the error of AdaBoost is $0 \%$, which agrees with reference ${ }^{26}$ published in our previous paper. However, the precision of AdaBoost is lower than that of MultiBoost with $k$-fold cross validation and testing data, which proofs poor robustness and over-fitting with full data set. This indicates MultiBoost algorithm is better.

\section{Discussion}

From the figures above, MultiBoost decision tree employed in classification is better than our previous work. Because the data set used in experiments is very small, we can't build a set of rules more accurately. Therefore, besides training and testing experiments, we also adopt a " $k$-fold cross validation" method to verify the validity of the method. It is worth noting that once the rules of decision tree are established, we can only use "if-else" rules to test new samples which can be implement easily for the embedded platforms.

Image processing is an effective method in classifying hydrophobic levels of insulators, and there are many image processing methods based on spraying images are proposed, such as WTH + EQU ${ }^{16}$ (White Top-Hat + Histogram Equalization), image segmentation with multi-threshold, ${ }^{18}$ etc. WTH+EQU is proposed by Thomazini et al., and they combine white top-hat, histogram equalization and sobel operator to obtain edges of droplets. In our experiments, we also adopt $\mathrm{WTH}+\mathrm{EQU}$ method to test their and our samples, and we get the same results with their images, but can't obtain satisfying results with our samples. It is because the samples are created with spraying solutions produced by mixtures of isopropyl alcohol and distilled water, and the solution presents a strong gray difference with background which is easy for segmentation. Furthermore, we conduct simple canny operator with their samples, and also get more accurate results which indicates the accuracy relies on their standard spraying images. Image segmentation with multi-threshold is applied in our previous work, and better results of images with uniform illumination can be obtained. Because of the transparency of droplets, there is little difference between backgrounds and droplets except edges. So we conclude that traditional image segmentation is not a valid method. Compared with our previous work, methods in this paper are more universally applied for uneven lighting images, but not for all the images (e.g. dirty insulators) and some parameters should be set manually (e.g. the size of structure element). Therefore, we will try to search for some adaptive methods in the following works. Furthermore, we apply other image segmentation methods, such as spectral clustering method, region growing algorithm, etc. ${ }^{34,35}$. But we can't obtain better results.

Four characteristic parameters adopted in this paper have specific geometric significance and synthesis some frequently-used characteristic parameters, such as circular factor, shape factor, ${ }^{9}$ cover rate, etc which can exclude the limitation of single parameter.

In classification, besides supervised and unsupervised clustering methods, we also applied mathematical regression method, such as PLS (Partial Least Squares), PCA (Principal Component Analysis) etc. But there is no satisfying nonlinear equation for prediction and we conclude that these four parameters have no obvious mathematical relation with $\mathrm{HC}$ levels.

Although AdaBoost algorithm get $0 \%$ error with full training data set, it is less accurate than MultiBoost. It indicates that AdaBoost is easy to be over trained and has lower generalization. Besides, SVM (support vector machine) applied in our previous work can also obtain good results. In the next following study, we want to search for some factors which can be expressed with equation like fractal dimension by Thomazini et al. ${ }^{19,20}$.

\section{Conclusion}

Measuring the hydrophobicity of insulated material's surface is important to supervise the quality of insulating 
material's production, and working insulators outdoors. In order to replace manual operation, we adopt image processing and pattern recognition method for classification.

We conduct many experiments with various analysis methods and finally decide to choose the above-mentioned method, "combine the canny operator and adaptive threshold using the integral image". The testing results are essentially satisfactory compared with our previous work (AdaBoost Decision tree). But the algorithm used for image processing is still complex and is only effective for most images, we will try to search for simple and more universal approaches and make them available on the embedded instrument.

We adopt four characteristic parameters to represent various hydrophobic levels which synthesize some merits proposed by other scholars. Given our previous work, we adopt a novel and simple method, MultiBoost decision tree,

\section{References}

1. Yuan J, Zhang JR, Wu JF, Duan NX, Qiu YC and Guo J. Analysis of hydrophobicity of composite insulators. Insulating Materials. 2002; 2:20-22.

2. Hackam R. Outdoor HV composite polymeric insulators. IEEE Transactions on Dielectrics and Electrical Insulation. 1999; 6(5):557-585. http://dx.doi.org/10.1109/94.798114.

3. Gorur RS. Status assessment of composite insulators for outdoor HV applications. In: Proceedings of the 5th International Conference on Properties and Applications of Dielectric Materials. Seoul, Korea: Korean Instifute of Elecitrical and Electronic Material Engineers; 1997. p. 35-38.

4. International Electrotechnical Commission. IEC62072 Guidance on the measurement of wettability of insulator surfaces. Geneva; 2003.

5. Berg M, Thottappillil R and Scuka V. Hydrophobicity estimation of HV polymeric insulating materials: Development of a digital image processing method. IEEE Transactions on Dielectrics and Electrical Insulation. 2001; 8(6):1098-1107. http://dx.doi.org/10.1109/94.971470.

6. Swedish Transmission Research Institute - STRI. Guide 92/1 Hydrophobicity Classification Guide; 1992.

7. Hartings R. Hydrophobicity of composite insulators: measurement and influence on flashover performance. In: Proceedings of the Stockholm Power Tech: International Symposium on Electric Power Engineering. New York, USA: Royal Institute of Technology; 1995. p. 246-251.

8. Gubanski S and Hartings R. Swedish research on the application of composite insulators in outdoor insulation. Electrical Insulation Magazine. 1995; 11(5):24-31. http:// dx.doi.org/10.1109/57.466414.

9. Tokoro T, Nagao M and Kosaki M. Image analyses of hydrophobicity of silicon rubber insulator. In: Annual Report Conference on Electrical Insulation and Dielectric Phenomena. Texas, USA:Institute of Electrical and Electronics Engineers; 1992. p. 763-766.

10. Altafim RAC, Santana AM, Murakami CR, Basso HC, Chierice GO and Neto SC. Hydrophobicity of polyurethane resins. In: Proceedings of the International Conference on Solid Dielectric. Toulouse, France: Institute of Electrical and Electronics Engineers; 2004. p. 5-9. http://dx.doi.org/10.1109/ ICSD.2004.1350388. to improve the performance of classification. MultiBoost decision tree can be used to reduce errors by combining the advantages of AdaBoost and Bagging. Furthermore, when the training process is completed, we can obtain the rules of classification. Then we can apply the "if-else" rules for testing without primary algorithm which lay a solid foundation for embedded implementation.

\section{Acknowledgments}

The authors acknowledge the assistance of WHVRI which provides SIR samples and the site for the experiments in this study. Furthermore, the authors also thank for the help of the 5th author, vice-professor Wang. With his early theories, the authors can put forward improvement programs and obtain better results.

11. Zhang R, Dong FM and Gao Z. Research on bead image recognition algorithm for determination of insulator levers. In: Proceedings of the International Conference on Computer Science and Information Technology. Beijing, China: Institute of Electrical and Electronics Engineers; 2009. p. 308-311.

12. Berg M, Thottappillil $\mathrm{R}$ and Scuka V. A digital image processing method for estimating the level of hydrophobicity of high voltage polymer. In: Proceedings of the Annual Report Conference on Electrical Insulation and Dielectric Phenomena. Texas, USA: Institute of Electrical and Electronics Engineers; 1999. p. 756-762.

13. Zhao L, Li C, Xiong J, Zhang S, Yao J and Chen X. Online hydrophobicity measurement for silicone rubber insulators on transmission lines. IEEE Transactions on Power Delivery. 2009; 24(2):806-813. http://dx.doi.org/10.1109/ TPWRD.2008.2005654.

14. Li Z, Liang X, Zhou Y, Tang J, Cui J and Liu Y. Influence of temperature on the hydrophobicity of silicone rubber surfaces. In: Proceedings of the Annual Report Conference on Electrical Insulation and Dielectric Phenomena. Colombia: Institute of Electrical and Electronics Engineers; 2004. p. 679-682.

15. Liang C, Yang WM and Liao QM. Water droplets segmentation for hydrophobicity classification. In: Proceedings of the International Conference on Acoustics, Speech and Signal Processing. Kyoto, Japan: Institute of Electrical and Electronics Engineers; 2012. p. 1181-1184.

16. Thomazini D, Gelfuso MV and Altafim RAC. Classification of polymers insulators hydrophobicity based on digital image processing. Materials Research. 2012; 15(3):365-371. http:// dx.doi.org/10.1590/S1516-14392012005000032.

17. Wang QD, Zhong ZF and Wang XP. Design and implementation of insulators material hydrophobicity measure system by support vector machine decision tree learning. In: Proceedings of the International Conference on Machine Learning and Cybernetics. Guangzhou, China: Institute of Electrical and Electronics Engineers; 2005. p. 4328-4334.

18. Wang QD, Wen BX and Wang XP. Measuring insulating material hydrophobic level by image recognition and classification. Electric Machines and Control. 2008; 12(1):9398.

19. Thomazini D, Gelfuso MV and Altafim RAC. Hydrophobicity classification of polymeric materials based on fractal 
dimension. Materials Research. 2008; 11(4):415-419. http:// dx.doi.org/10.1590/S1516-14392008000400006.

20. Thomazini D, Gelfuso MV and Altafim RAC. Analysis of entropy and fractal dimension descriptors to classify the hydrophobicity in polymeric insulators. In: Proceedings of the International Symposium on Electrical Insulating. Mie, Japan; 2008. p. 279-282.

21. Tokoro T, Iwasaki T and Kosaki M. Diagnosis of hydrophobic condition of polymer materials using dielectric measurement and image analysis. In: Proceedings of the Annual Report Conference on Electrical Insulation and Dielectric Phenomena. Colombia: Institute of Electrical and Electronics Engineers; 2004. p. 627-630

22. Qi B, Tang LR and Zhang J. Research on measurement of hydrophobicity of insulators. Proceedings of the Chinese Society for Electrical Engineering. 2008; 28(31):120-124.

23. Freund $Y$ and Schapire RE. A decision-theoretic generalization of on-line learning and an application to boosting. Journal of Computer and System Sciences. 1997; 55(1):119-139. http:// dx.doi.org/10.1006/jcss.1997.1504.

24. Breiman L. Bagging predictors. Machine Learning. 1996; 24(2):123-140. http://dx.doi.org/10.1007/BF00058655.

25. Webb GI. MultiBoosting: a technique for combining boosting and wagging. Machine Learning. 2000; 40(2):159-196. http:// dx.doi.org/10.1023/A:1007659514849.

26. Bernsen J. Dynamic thresholding of gray-level images. In: Proceedings of the International Conference on Pattern Recognition. Paris, France; 1986. p. 1251-1255.

27. Wellner P. Adaptive thresholding for the digitaldesk. Cambridge: Xerox Research Centre; 1993. Technical Report EPC-93-110, EuroPARC.
28. Bradley D and Roth G. Adaptive thresholding using the integral image. Journal of Graphics, GPU and Game Tools. 2007; 12(2):13-21. http://dx.doi.org/10.1080/215123 7X.2007.10129236.

29. Canny J. A computational approach to edge detection. IEEE Transactions on Pattern Analysis and Machine Intelligence. 1986; PAMI-8(6):679-698. http://dx.doi.org/10.1109/ TPAMI.1986.4767851. PMid:21869365

30. Gao J and Liu N. An improved adaptive threshold canny edge detection algorithm. In: Proceedings of the International Conference on Computer Science and Electronic Engineering. Hangzhou, China: Institute of Electrical and Electronics Engineers; 2012. p. 164-168.

31. Haralick RM, Sternberg SR and Zhuang X. Image analysis using mathematical morphology. IEEE Transactions on Pattern Analysis and Machine Intelligence. 1987; PAMI-9(4):532550. http://dx.doi.org/10.1109/TPAMI.1987.4767941. PMid:21869411

32. Peng KX, Wang QD and Wang XP. Spray image analysis based measurement of hydrophobic of insulator surfaces. Insulating Material. 2005; 1:47-51.

33. Wold S, Kettaneh-Wold N and Skagerberg B. Nonlinear PLS modeling. Chemometrics and Intelligent Laboratory Systems. 1989; 7(1-2):53-65. http://dx.doi.org/10.1016/01697439(89)80111-X.

34. von Luxburg U. A tutorial on spectral clustering. Statistics and Computing. 2007; 17(4):395-416. http://dx.doi.org/10.1007/ s11222-007-9033-z.

35. Sumuya, Gao C and Chai S. A note on spectral clustering method based on normalized cut criterion. In: Proceedings of the Chinese Conference on Pattern Recognition. Nanjing, China: Institute of Electrical and Electronics Engineers; 2009. p. 1-5. http://dx.doi.org/10.1109/CCPR.2009.5343984. 